\title{
Research Paper: \\ Incidence and Outcomes of Acute Lung Injury in Patients With Isolated Traumatic Brain Injury
}

\author{
Siamak Rimaz ${ }^{1}$, Seyyed Mahdi Zia Ziabari ${ }^{2}$, Neshat Jabbari ${ }^{1} Q$, Zahra Pourmohammadi ${ }^{3}$, Parrisa Bagheri ${ }^{4} Q$, Sheyda Rimaz ${ }^{5^{*}}$
}

1. Guilan Road Trauma Research Center, Guilan University of Medical Sciences, Rasht, Iran

2. Department of Emergency Medicine, School of Medicine, Guilan University of Medical Sciences, Rasht, Iran

3. Burn and Regenerative Medicine Research Center, Guilan University of Medical Sciences, Rasht, Iran

4. Health Information Management Research Center, Kashan University of Medical Sciences, Kashan, Iran

5. Student Research Committee, School of Medicine, Anzali International Campus, Guilan University of Medical Sciences, Rasht, Iran

\begin{tabular}{|l|l|}
$\begin{array}{c}\text { Use your device to scan } \\
\text { and read the article online }\end{array}$ & $\begin{array}{l}\text { Cittation Rimaz S, Zia Ziabari SM, Jabbari N, Pourmohammadi Z, Bagheri P, Rimaz S. Incidence and Outcomes of Acute } \\
\text { Lung Injury in Patients With Isolated Traumatic Brain Injury. Iran J Neurosurg. 2021; 7(3):139-146. http://dx.doi.org/10.32598/ } \\
\text { irjns.7.3.3 }\end{array}$ \\
d.t:: http://dx.doi.org/10.32598/irjns.7.3.3
\end{tabular}

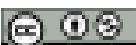

Article info:

Received: 20 Apr 2021

Accepted: 11 Jun 2021

Available Online: 01 Jul 2021

Keywords:

Acute lung injury, Traumatic brain injury, Glasgow Coma Scale (GCS)

\section{A B S T RACT}

Background and Aim: Traumatic Brain Injury (TBI) is an essential cause of morbidity and mortality worldwide. TBI patients frequently encounter lung complications, such as Acute Lung Injury (ALI) and Acute Respiratory Distress Syndrome (ARDS), which is associated with poor clinical outcome because hypoxia causes additional injury to the brain. This study aimed to evaluate the frequency of $\mathrm{ALI}$ in patients with $\mathrm{TBI}$ and its consequences.

Methods and Materials/Patients: In this descriptive cross-sectional study, data from all records of patients admitted to Poursina Hospital's ICU (emergency and neurosurgery ICU) in 20 18-2019 were used. The evaluated data included age, gender, type of head trauma mechanism, kind of brain injury based on CT scan findings, the severity of brain injury based on Glasgow Coma Scale (GCS), underlying diseases, mean head AIS score, the number of pack cell units injected, as well as bilateral pulmonary infiltration in favor of ALI and brain injury.

Results: Only 81 of the 557 TBI cases met the inclusion criteria of the present study. The highest frequency of ALI following TBI was observed on the first day of hospitalization, in men $(0.41 \%)$ in the age group of $40-50$ years $(7 \%)$ with severe brain damage $(6 \%)$ and subdural hematoma (12\%), following a motorcycle accident, cars, as well as on the third day of hospitalization were seen in men (43.8\%) with the age group of $20-30$ years (55\%) with severe brain damage (42\%) and intra-parenchymal bleeding (57\%), following a motorcycle accident. In addition, no significant correlation was detected between the incidence of ALI and mortality, the duration of hospitalization, GCS, mean head AIS score, or the extent of received blood units in our study.

Conclusion: According to the obtained findings, men aged between 20 and 30 years with severe cerebral injury, epidural hematoma and a motorcycle accident presented the highest rate of progression toward $\mathrm{ALI}$ in the first to third days of hospitalization.

* Corresponding Author:

Sheyda Rimaz, MD.

Address: Student Research Committee, School of Medicine, Anzali International Campus, Guilan University of Medical Sciences, Rasht, Iran Tel: +98 (911) 8063735

E-mail: rimazsh@ymail.com 


\section{Highlights}

- Acute Lung Injury (ALI) is relatively common after Traumatic Brain Injury (TBI) and 15\% of patients with isolated moderate to severe TBI developed ALI.

- The frequency of patients who progressed to acute lung injury by the third day of hospitalization increased, compared to that of the first day. Moreover, the highest frequency respected the age group of 20-30 years (55.1\%).

- The obtained results signified that on the third day, the highest frequency of acute lung injury concerned epidural hematoma (52\%), Subdural Hematoma (SDH) (36\%), and contusion (35.2\%).

- The development of ALI is a critical independent factor affecting mortality in patients with traumatic brain injury, although this difference was not significant.

\section{Plain Language Summary}

Pulmonary complications, such as Acute Lung Injury (ALI) occur in $20 \%-25 \%$ of patients with isolated brain injury and have a poor prognosis. The present study aimed to determine the incidence of ALI in trauma patients who were admitted to Poursina Hospital. We aimed to assess the impact of ALI on mortality after isolated traumatic brain injury. Moreover, we intended to identify whether the development of ALI correlates with the severity of Traumatic Brain Injury (TBI). For this purpose, clinical data were collected in 2 years at Poursina Hospital. Our results addressed the highest frequency of ALI following TBI on the first day of hospitalization, in men (0.41\%) in the age group of 40-50 years (7\%) with severe brain damage $(6 \%)$ and subdural hematoma (12\%) following a motorcycle-car accident; on the third day of hospitalization, they were detected in men (43.8\%) in the age group of 20-30 years (55\%) with severe brain damage (42\%) and epidural hematoma (52\%), following a motorcycle accident. Besides, no significant correlation was observed between the incidence of ALI and mortality, the duration of hospitalization, Glasgow Coma Scale (GCS), mean head AIS score, or the extent of received blood units. The development of ALI is a critical independent factor affecting mortality in patients with TBI. According to the present study findings, men aged 20-30 years with severe cerebral injury, intra-parenchymal hemorrhage, and a motorcycle accident presented the highest rate of progression toward ALI during the first to third days of hospitalization.

\section{Introduction}

cute severe Traumatic Brain Injury (TBI) A is among the world's most serious concerns, as well as a leading cause of disability and mortality [1-4]. TBI may cause complications in other organs, like the lungs in addition to direct brain damage, resulting in systemic inflammatory factors [5]. Damaged cells secrete a slew of inflammatory mediators, including cytokines, chemokines, and Danger-Associated Molecular Patterns (DAMPS). Moreover, they contribute to the inflammatory process in the brain and other distant organs, including the lungs [6]. Acute Lung Injury (ALI) and its more severe form, Acute Respiratory Distress Syndrome (ARDS), are followed by an inflammatory pulmonary response characterized by severe hypoxemia, reduced pulmonary compliance, and pulmonary infiltration induced by direct or indirect lung tissue damage [7].
Despite significant advances in understanding the pathophysiology of the aforementioned disease, there exists substantial morbidity and mortality [8]. Mechanical ventilation is used as a mechanism to ensure reliable oxygen delivery and modulate cerebral hemodynamics through the control of arterial carbon dioxide tension. Concurrently, mechanical ventilation can exert harmful effects on the brain due to complex physiological interactions between intrathoracic, central venous, and intracranial compartments $[9,10]$. In patients with acute $\mathrm{TBI}$, the incidence of $\mathrm{ALI}$ and ARDS was reported in some studies to be around 30\% [6]; in patients with isolated head trauma, the incidence was reported to range between $20 \%$ and $25 \%$. Moreover, this complication being an independent predictor factor for poor prognosis. This data discrepancy was caused by differences in the inclusion criteria; regardless of these differences, all of these studies reported that the presence of ALI/ARDS was associated with increased mortality and morbidity in patients with severe brain injury [11-18]. It was also associated 
with prolonged hospitalization in the ICU and hospital, as well as reduced days without ventilators [14].

The most recent definition of ARDS presented in 1994 in the American-European Conference Consensus Committee [7] is an acute onset syndrome, bilateral pulmonary infiltration in chest radiography, pulmonary edema, pulmonary artery wedge pressure less than $18 \mathrm{mmHg}$, or the lack of clinical signs of high left atrial pressure, and hypoxia with a ratio of $\mathrm{PaO} 2 / \mathrm{FiO} 2<200$, patients who meet the above criteria; however, have Pao2/Fio2<300 will generate ALI diagnosis. The severity of brain damage based on brain CT scan findings, as well as a low level of consciousness (Glasgow Coma Scale, GCS), were identified as risk factors for progression to ALI/ARDS in preliminary studies [11, 12]. However, additional cranial characteristics, such as using vasoactive drugs, a history of drug abuse, lung contusion, and multiple transfusions have recently been proposed [12]. Thus, the current study aimed to determine the incidence of $\mathrm{ALI}$ in patients with severe isolated TBI. Moreover, we evaluated the impact of ALI on mortality and neurologic outcome after severe TBI. Besides, we aimed to identify whether the development of ALI correlates with the severity of TBI.

\section{Methods and Materials/Patients}

This was a prospective, analytical, and cross-sectional study. The necessary data were collected in 2-years with the permission of the patient or patient's family and with the approval of the Guilan University of Medical Sciences Ethics Committee (Code: GUMS.REC.1397.236) in the Poursina Medical Research and Training Hospital Center in Rasht City, Iran, in 2018-2019. Clinical data were collected on arrival to the emergency department, and a daily basis during the Neurosurgery Intensive Care Unit (N-ICU) stay. The inclusion criteria included patients aged between 18 and 55 years, having isolated head trauma with a head AIS score of $\geq 3$ (determined by the Head Abbreviated Injury Scale), no other body region AIS score of $\geq 3$, and receiving supplemental oxygen via a face mask, $t$-tube, or mechanical ventilation for $>24$ hours after hospitalization. The study patients underwent endotracheal intubation and mechanical ventilation if they had a combination of the following factors, including the level of consciousness (GCS $\leq 8$ ), severe agitation and combativeness, the loss of airway protective reflexes, significant Intracranial Pressure (ICP) elevation, as well as hypoxemic respiratory failure, i.e., refractory to conventional supplemental oxygen.
The file with incomplete information was excluded from the study. The demographic information of the study patients, including age, gender, underlying disease, the level of consciousness at the hospital according to Glasgow criteria, the type of mechanism of head trauma (fall, accident, etc.), the type of brain injury based on CT scan findings (epidural hematoma, subdural hematoma, intra-parenchymal hemorrhage, intraventricular hemorrhage, diffuse axonal injury \& cerebral edema, contusion, cranial fracture, \& pneumocephalus).

The severity of TBI was stratified by grouping the research patients according to GCS score [3-8, 11-17], mean AIS head score, the number of pack cell units transfused in the first 24 hours of hospitalization, the type of treatment (surgery or conservative treatment), the type of airway management (intubation or nonintubation), the duration of hospitalization, and GCS at the time of discharge, as well as the patient outcome as improvement or in-hospital death was assessed and recorded. The diagnosis of ALI was based on the International Consensus Criteria per $\mathrm{PaO} 2 / \mathrm{FiO} 2$ ratio on the first and third days. If $\mathrm{PaO} 2 / \mathrm{FiO} 2$ equaled $<300$, a chest $\mathrm{X}$-ray was performed on the same day. Furthermore, according to a radiologist report (who was blinded to the patient's clinical course) on bilateral pulmonary infiltration, ALI was diagnosed [19]. The obtained data were analyzed by SPSS software. For descriptive findings, mean, standard deviation, and odds ratio were applied. The single logistic regression test investigated the relationship between acute pulmonary injury and brain injury outcome.

\section{Results}

In total, the records of 557 patients with traumatic brain injury were reviewed in this study. Moreover, 81 patients met the inclusion criteria. Accordingly, $15 \%$ of patients with isolated moderate to severe TBI developed ALI. Of these, $74(91.35 \%)$ subjects were male, and $7(8.64 \%)$ were female. The Mean $\pm S D$ age of the study subjects was $32.82 \pm 11.25$ years. The relevant results revealed that the frequency of patients who progressed to ALI by the third day of hospitalization increased, compared to the first day. Moreover, the highest frequency belonged to the age group of $20-30$ years (55.1\%). Finally, the lowest rate concerned the age group $50-60$ years (10\%).

Regarding the frequency of ALI respecting gender, $43.8 \%$ of men and $12.5 \%$ of women presented $A L I$ on the third day. Examining the frequency of ALI based on the severity of brain injury suggested that on the first day of hospitalization, only in the group of patients with severe brain injury (GCS=3-8), 6\% developed ALI. 
Table 1. Relationship between acute lung injury and demographic and treatment variables of study patients

\begin{tabular}{|c|c|c|c|c|c|}
\hline Variables & OR & SD & $\mathbf{Z}$ & $\mathbf{P}$ & Min. to Max. \\
\hline Airway intubation & 0.923 & 0.42 & -0.17 & 0.86 & $2.29-0.371$ \\
\hline $\begin{array}{l}\text { Surgical treatment } \\
\text { (Craniotomy) }\end{array}$ & 3.29 & 1.56 & 2.51 & 0.012 & 8.1-34.301 \\
\hline Brain injury outcome & 1.28 & 0.515 & -0.49 & 0.626 & $3.0-53.468$ \\
\hline Duration of hospitalization & 1.093 & 0.052 & 1.70 & 0.088 & $1.0-21.986$ \\
\hline Number of packed cells transfused & 0.895 & 0.317 & -0.35 & 0.727 & $0.511-0.733$ \\
\hline Head AIS score & 0.942 & 0.417 & -0.84 & 0.400 & $1.190-0.746$ \\
\hline GCS at discharge time & 1.194 & 0.497 & 0.43 & 0.668 & $2.691-0.530$ \\
\hline
\end{tabular}

Within each dependent measure, means with different subscripts differ significantly $(P<0.05)$; OR: Odds Ratio; SD: Standard Deviation; Z: Z Statistics; P: P-value.

However, on the third day of hospitalization, respecting moderate injury (GCS=9-12) and severe brain injury (GCS=3-8), respectively, $38.7 \%$ and $42 \%$ of the study subjects generated ALI. The collected results manifested that most brain findings were related to contusion based on CT scan findings; the lowest respected pneumocephalus and hydrocephalus. On the third day, the highest frequency of ALI was related to epidural hematoma (52\%), subdural hematoma (SDH) (36\%), and contusion (35.2\%). On the first day, the most common ALI was reported with subdural hematoma (12\%). Concerning underlying diseases, the most common comorbid condition was hypertension in the study population. Furthermore, $66 \%$ of patients who had endotracheal intubation on the first day and $6 \%$ on the third day had acute lung injury, i.e., not much different from those who had no intubation $(61 \%$ $\& 62 \%$ on the first \& third day, respectively).

In terms of treatment, $66.6 \%$ of patients who underwent craniotomy had ALI on the first day, while $39.7 \%$ of patients undergoing conservative treatment presented $\mathrm{ALI}$ in the same period. This frequency was reported to be $57.5 \%$ vs. $29.1 \%$ on the third day in patients who underwent craniotomy and maintenance treatment, respectively. Table 1 presents the relationship between ALI and airway intubation, surgical treatment, brain injury outcome, the duration of hospitalization, the number of packed cells, head AIS score, and GCS at discharge time. Table 1 presents that craniotomy alone was directly and significantly associated with ALI.

Table 2 examines the relationship between the difference between ALI on the first and third day based on GCS upon arrival. This table outlines that the coefficient of relationship between these two variables equaled 127.81, indicating a direct relationship between these variables; however, it was not statistically significant $(P=0.210)$.

\section{Discussion}

The present study determined the incidence of $A L I$ in trauma patients with severe $\mathrm{TBI}$; evaluated the impact of ALI on mortality and neurologic outcome after severe $\mathrm{TBI}$, and o identified whether the development of ALI correlates with the severity of TBI. The obtained results suggested that $15 \%$ of the examined patients with severe TBI developed ALI.

In this study, the highest incidence of ALI following TBI on the first day of hospitalization belonged to the age group of 40-50 years (7\%); on the third day of hospitalization, this rate belonged to the age group of $20-30$ years (55.1\%). In addition, in both periods, the incidence of men was higher than women $(0.41 \%$ vs. zero on the first day $\& 43.8 \%$ vs. $12.5 \%$ on the third day). In the study by Hendrickson et al., the mean age of patients with ARDS following TBI was equal to 45 years [20]. Furthermore, $87 \%$ of the patients were male. In the study of Scholz et al., the incidence of ARDS following TBI at admission was higher in the elderly; however, 48 hours later, there was no significant difference between age groups. Moreover, ARDS was higher in men than women in the first 6 hours and 18 hours after TBI [21].

The collected data revealed no significant gender-wise difference and the frequency of ALI on the first and third 
Table 2. Difference between acute lung injury on the first and third day by sex, age, trauma mechanism, and GCS on arrival

\begin{tabular}{|c|c|c|c|c|c|c|}
\hline Variables & Coefficient & SD & $\mathbf{t}$ & $\mathbf{P}$ & Min. & Max. \\
\hline Gender & -86.94 & 168.07 & -0.52 & -0.52 & -421.69 & 247.802 \\
\hline Age & -6.059 & 4.614 & -1.31 & -1.31 & -15.249 & 3.130 \\
\hline Mechanistic of trauma & 35.136 & 25.364 & 1.39 & 1.39 & -15.381 & 85.654 \\
\hline GCS upon arrival & 127.817 & 100.997 & 1.27 & 1.27 & -73.337 & 328.971 \\
\hline
\end{tabular}

Within each dependent measure, means with different subscripts differ significantly $(\mathrm{P}<0.05)$.

day of hospitalization; however, the risk of ALI following TBI in men was more than that in women. This may be due to the higher incidence of high-risk behaviors, like tobacco or alcohol use, among males.

The present study results indicated that in the first and third days of hospitalization, the highest incidence of ALI following TBI concerned individuals with severe brain injury (GCS=3-8) ( $6 \%$ on the first day \& $42 \%$ on the third day). However, no patient with a mild brain injury presented ALI. Moreover, there was no significant difference between the severity of brain injury and the frequency of ALI on the first and third days of hospitalization (moderate vs. severe TBI). In the study by Hendrickson et al. [20], the mean GCS in patients with ARDS following TBI at admission was measured to be 6, i.e., lower than that in those without ARDS (GCS=7). In other words, the severity of brain damage in individuals with pulmonary complications following traumatic brain injury was higher than that in those without these complications. However, in the study of Kazemi et al., the Mean \pm SD GCS score in patients with TBI-induced ARDS (9.4 \pm 4.9$)$ was significantly higher than that in those without this syndrome $(5.6 \pm 3.2)$ [22].

In our study, the highest incidence of ALI was reported on the first day of hospitalization in patients with SDH $(12 \%)$ and Subarachnoid hemorrhage (SAH) (10\%). These statistics concerned epidural hematoma (52\%), SDH $(36 \%)$, and contusion (35.2\%) on the third day of hospitalization. In the study of Kazemi et al., the most common complication associated with TBI in patients with ARDS were subdural hematoma (57.1\%), cerebral contusion $(51.4 \%)$, and spider hemorrhage (40\%), respectively. In the study of the history of underlying diseases, 6 subjects in the present investigation had hypertension, only one of whom presented ALI on the third day. In Kazemi et al.'s [22] study, hypertension was the most frequent underlying disease associated with ARDS in patients with TBI. These results were in line with those of Contant and colleagues [12]. In this study, hypertension pressure was also suggested as a risk factor for ARDS following TBI.

In our study, no significant relationship was found between the incidence of $A L I$ and death, intubation, hospital stay, blood transfusion, mean head AIS score, and the GCS of patients at discharge. Although the incidence of $\mathrm{ALI}$ was directly related to the rate of intubation, mortality, hospital stay, and the GCS of patients at discharge; it was inversely related to the units of received blood units, the mean AIS score was on their heads. There was also a significant relationship between the incidence of ALI on the third day and craniotomy. In the study by Lee et al. [23], in line with our study, an increase in mortality and mortality in patients with TBI-induced ARDS was reported. Robba et al. [24] also stated that acute respiratory distress syndrome, acute pulmonary injury, is a strong risk factor for in-hospital death and mortality in patients with traumatic brain injury. In the study of Hendrickson et al. [20], contrary to the results of our study, a significant relationship was found between ALI and AIS head scores. In Hendrickson's study [20], the relationship between $\mathrm{ALI}$ and the number of received blood units by patients was not studied; however, no significant difference was found between patients with ARDS following $\mathrm{TBI}$ and those without it concerning the number of received blood units.

\section{Conclusion}

TBI-Induced ALI is a relatively common complication. The present research results suggested that this complication would occur more on the third day. Factors, such as severe brain damage, the age range of 20 to 30 years, and epidural hematoma are risk factors for this complication. Attention to this complication and precise respiratory care should be considered in ALI patients. Further studies are necessary to create diagnostic and therapeutic guidelines that can provide a better clinical outcome for these patients. 


\section{Ethical Considerations}

Compliance with ethical guidelines

This article was approved by the institutional review board of Guilan University of Medical Sciences (Code: IR.GUMS.REC.1397.236). The necessary data were collected with the permission of the patient or the patient's family.

Funding

This article was extracted from a thesis which was defended at Guilan University of Medical Sciences, Rasht (Code: GUMS.REC.1397.236).

\section{Authors' contributions}

All authors equally contributed to preparing this article.

Conflict of interest

The authors declared no conflict of interest.

\section{References}

[1] Bakowitz M, Bruns B, McCunn M. Acute lung injury and the acute respiratory distress syndrome in the injured patient. Scandinavian Journal of Trauma, Resuscitation and Emergency Medicine. 2012; 20:54. [DOI:10.1186/1757-7241-20-54] [PMID] [PMCID]

[2] Della Torre V, Badenes R, Corradi F, Racca F, Lavinio A, Matta $\mathrm{B}$, et al. Acute respiratory distress syndrome in traumatic brain injury: How do we manage it? Journal of Thoracic Disease. 2017; 9(12):5368-81. [DOI:10.21037/jtd.2017.11.03] [PMID] [PMCID]

[3] Rakib F, Al-Saad K, Ahmed T, Ullah E, Barreto GE, Ashraf GM, et al. Biomolecular alterations in acute Traumatic Brain Injury (TBI) using Fourier Transform Infrared (FTIR) imaging spectroscopy. Spectrochimica Acta. Part A, Molecular and Biomolecular Spectroscopy. 2021; 248:119189. [DOI:10.1016/j. saa.2020.119189] [PMID]

[4] Asmamaw Y, Yitayal M, Debie A, Handebo S. The costs of traumatic head injury and associated factors at University of Gondar Specialized Referral Hospital, Northwest Ethiopia. BMC Public Health. 2019; 19(1):1399. [DOI:10.1186/s12889019-7800-3] [PMID] [PMCID]

[5] Rincon F, Ghosh S, Dey S, Maltenfort M, Vibbert M, Urtecho J, et al. Impact of acute lung injury and acute respiratory distress syndrome after traumatic brain injury in the United States. Neurosurgery. 2012; 71(4):795-803. [DOI:10.1227/ NEU.0b013e3182672ae5] [PMID]

[6] Nicolls MR, Laubach VE. Traumatic brain injury: Lungs in a RAGE. Science Translational Medicine. 2014; 6(252):252fs34. [DOI:10.1126/scitranslmed.3010259] [PMID] [PMCID]
[7] Bernard GR, Artigas A, Brigham KL, Carlet J, Falke K, Hudson L, et al. The American-European Consensus Conference on ARDS. Definitions, mechanisms, relevant outcomes, and clinical trial coordination. American Journal of Respiratory and Critical Care Medicine. 1994; 149(3):818-24. [DOI:10.1164/ajrccm.149.3.7509706] [PMID]

[8] Yadav H, Nolan ME, Bohman JK, Cartin-Ceba R, Peters SG, Hogan WJ, et al. Epidemiology of acute respiratory distress syndrome following hematopoietic stem cell transplantation Critical Care Medicine. 2016; 44(6):1082-90. [DOI:10.1097/ CCM.0000000000001617] [PMID] [PMCID]

[9] Piek J, Chesnut RM, Marshall LF, van Berkum-Clark M, Klauber MR, Blunt BA, et al. Extracranial complications of severe head injury. Journal of Neurosurgery. 1992; 77(6):901-7. [PMID] [DOI:10.3171/jns.1992.77.6.0901]

[10] Borsellino B, Schultz MJ, Gama de Abreu M, Robba C, Bilotta F. Mechanical ventilation in neurocritical care patients: a systematic literature review. Expert Review of Respiratory Medicine. 2016; 10(10):1123-32. [DOI:10.1080/17476348.2017.1235976 ] [PMID]

[11] Holland MC, Mackersie RC, Morabito D, Campbell AR, Kivett VA, Patel R, et al. The development of acute lung injury is associated with worse neurologic outcome in patients with severe traumatic brain injury. The Journal of Trauma. 2003; 55(1):106-11. [DOI:10.1097/01.TA.0000071620.27375.BE] [PMID]

[12] Contant CF, Valadka AB, Gopinath SP, Hannay HJ, Robertson CS. Adult respiratory distress syndrome: A complication of induced hypertension after severe head injury. Journal of Neurosurgery. 2001; 95(4):560-8. [DOI:10.3171/jns.2001.95.4.0560] [PMID]

[13] Bratton SL, Davis RL. Acute lung injury in isolated traumatic brain injury. Neurosurgery. 1997; 40(4):707-12 [DOI:10.1097/00006123-199704000-00009] [PMID]

[14] Mascia L, Zavala E, Bosma K, Pasero D, Decaroli D, Andrews $\mathrm{P}$, et al. High tidal volume is associated with the development of acute lung injury after severe brain injury: An international observational study. Critical Care Medicine. 2007; 35(8):1815-20. [DOI:10.1097/01.CCM.0000275269.77467.DF] [PMID]

[15] Kahn JM, Caldwell EC, Deem S, Newell DW, Heckbert SR, Rubenfeld GD. Acute lung injury in patients with subarachnoid hemorrhage: Incidence, risk factors, and outcome Critical Care Medicine. 2006; 34(1):196-202. [DOI:10.1097/01. CCM.0000194540.44020.8E] [PMID]

[16] Goyal K, Hazarika A, Khandelwal A, Sokhal N, Bindra A Kumar N, et al. Non-neurological complications after traumatic brain injury: A prospective observational study. Indian Journal of Critical Care Medicine. 2018; 22(9):632-8. [DOI:10.4103/ijccm. IJCCM_156_18] [PMID] [PMCID]

[17] Zygun DA, Kortbeek JB, Fick GH, Laupland KB, Doig CJ. Non-neurologic organ dysfunction in severe traumatic brain injury. Critical Care Medicine. 2005; 33(3):654-60. [DOI:10.1097/01. CCM.0000155911.01844.54] [PMID]

[18] Solenski NJ, Haley ECJ, Kassell NF, Kongable G, Germanson $\mathrm{T}$, Truskowski L, et al. Medical complications of aneurysmal subarachnoid hemorrhage: A report of the multicenter, cooperative aneurysm study. Critical Care Medicine. 1995; 23(6):1007-17 [DOI:10.1097/00003246-199506000-00004] [PMID]

[19] Evans TW. International Consensus Conferences in Intensive Care Medicine: non-invasive positive pressure ventilation in acute respiratory failure. Organised jointly by the American Thoracic Society, the European Respiratory Society, the European Society of Intensive Care Medicine, and the Sociéte de 
Réanimation de Langue Française, and approved by the ATS Board of Directors, December 2000. Intensive Care Medicine. 2001; 27(1):166-78. [DOI:10.1007/s001340000721] [PMID]

[20] Hendrickson CM, Howard BM, Kornblith LZ, Conroy AS, Nelson MF, Zhuo H, et al. The acute respiratory distress syndrome following isolated severe traumatic brain injury. The Journal of Trauma and Acute Care Surgery. 2016; 80(6):989-97. [DOI:10.1097/TA.0000000000000982] [PMID] [PMCID]

[21] Scholz M, Cinatl J, Schädel-Höpfner M, Windolf J. Neutrophils and the blood-brain barrier dysfunction after trauma. Medicinal Research Reviews. 2007; 27(3):401-16. [DOI:10.1002/med.20064] [PMID]

[22] Kazemi S, Hosseinabad MS, Ameri M, Ghorbani Yekta B. [Acute respiratory distress syndrome assessment after traumatic brain injury (Persian)]. Tehran University Medical Journal. 2016; 73(10):739-43. http://tumj.tums.ac.ir/article-1-7088-en.html

[23] Lee K, Rincon F. Pulmonary complications in patients with severe brain injury. Critical Care Research and Practice. 2012; 2012:207247. [DOI:10.1155/2012/207247] [PMID] [PMCID]

[24] Robba C, Asgari S, Gupta A, Badenes R, Sekhon M, Bequiri E, et al. Lung injury is a predictor of cerebral hypoxia and mortality in traumatic brain injury. Frontiers in Neurology. 2020; 11:771. [DOI:10.3389/fneur.2020.00771] [PMID] [PMCID] 
This Page Intentionally Left Blank 